\title{
Long non-coding RNA H19 mediates N-acetyltransferase 1 gene methylation in the development of tamoxifen resistance in breast cancer
}

\author{
HONG SUN ${ }^{1}$, GUO WANG $^{2-5}$, JIAQIN CAI ${ }^{1}$, XIAOXIA WEI ${ }^{1}$, \\ YING ZENG ${ }^{2}$, YAN PENG ${ }^{2}$ and JIE ZHUANG ${ }^{1}$ \\ ${ }^{1}$ Department of Pharmacy, Shengli Clinical Medical College of Fujian Medical University, Fujian \\ Provincial Hospital, Fuzhou, Fujian 350001; ${ }^{2}$ Department of Clinical Pharmacology, Xiangya Hospital, \\ Central South University; ${ }^{3}$ Institute of Clinical Pharmacology, Central South University; ${ }^{4}$ Hunan Key \\ Laboratory of Pharmacogenetics, Changsha, Hunan 410078; ${ }^{5}$ National Clinical Research Center for Geriatric \\ Disorders, Xiangya Hospital, Central South University, Changsha, Hunan 410008, P.R. China
}

Received September 23, 2020; Accepted July 7, 2021

DOI: $10.3892 / \mathrm{etm} .2021 .10934$

\begin{abstract}
Long non-coding RNA (lncRNA) H19 is associated with proliferation, invasion and metastasis in numerous types of cancer. H19 lncRNA has been demonstrated to be an estrogen-inducible gene, the expression of which is significantly increased in tamoxifen (TAM)-resistant MCF-7 breast cancer cells. The aim of the present study was to investigate the role and molecular mechanism of lncRNA H19 in the development of TAM resistance. TAM-resistant MCF-7 (MCF-7R) cells were developed by the treatment of wild-type MCF-7 cells with 4-hydroxytamoxifen. Analysis of H19 expression in the cells indicated that upregulation of H19 contributed to the resistance of the MCF-7R cell line. Furthermore, when H19 was knocked down in the MCF-7R cells, the sensitivity to 4-hydroxytamoxifen was markedly restored. The results further demonstrated that $\mathrm{N}$-acetyltransferase 1 (NAT1) may serve an important role in TAM-resistant cells, as NAT1 expression was notably downregulated in the MCF-7R cells but significantly elevated following the knockdown of H19. In addition, lower expression of NAT1 and higher expression of H19 were indicated to be associated with poor prognosis in patients with breast cancer treated with TAM. The results of
\end{abstract}

Correspondence to: Professor Jie Zhuang, Department of Pharmacy, Shengli Clinical Medical College of Fujian Medical University, Fujian Provincial Hospital, 134 Dongjie Street, Fuzhou, Fujian 350001, P.R. China

E-mail: zhuangjie2019@163.com

Professor Guo Wang, Department of Clinical Pharmacology, Xiangya Hospital, Central South University, 110 Xiangya Road, Changsha, Hunan 410078, P.R. China

E-mail: 207082@csu.edu.cn

Key words: drug resistance, long non-coding RNA H19, $\mathrm{N}$-acetyltransferase 1, DNA methylation, breast cancer bisulfite genomic sequencing PCR analysis indicated that the methylation rate of NAT1 in MCF-7R cells was significantly higher compared with that in MCF-7 cells, while the methylation rate of NAT1 in TAM-resistant cells transfected with small interfering RNA against H19 was significantly lower than that in the corresponding untransfected cells. Therefore, the present study suggests that the H19 gene regulates NAT1 expression in TAM-resistant cells via the mediation of NAT1 promoter methylation.

\section{Introduction}

Breast cancer is the most frequently diagnosed type of cancer in women and is the leading cause of cancer-associated mortality in women worldwide (1). In total, $\geq 70 \%$ of breast cancer cases are classified as estrogen receptor (ER) $\alpha$-positive breast cancer (2). Tamoxifen (TAM), a partial ER agonist, is the gold standard first-line endocrine therapy for premenopausal patients with ER $\alpha$-positive breast cancer. Furthermore, the adjuvant therapy of breast cancer with TAM for $\sim 5$ years can reduce the rate of disease recurrence by one-half and annual breast cancer mortality by one-third (3). Despite these clear benefits, resistance to TAM therapy is a challenge, and $\sim 40 \%$ of patients with ER $\alpha$-positive breast cancer eventually develop TAM resistance (4). It has been revealed that the epigenetic regulation of gene expression is a major cause of TAM resistance in the laboratory and clinic (5). The methylation of $\mathrm{CpG}$ sites in DNA promoter sequences is an important epigenetic mechanism that may result in the transcriptional inactivation of genes and the modulation of drug resistance in breast cancer (6). There is evidence to suggest that TAM resistance is associated with the methylation and expression of the N-acetyltransferase 1 (NAT1) gene $(7,8)$. However, the molecular mechanism involved in the regulation of NAT1 gene expression and methylation remains unknown.

Long non-coding RNA (lncRNA) H19 is an imprinted oncofetal gene (9). The expression level of lncRNA H19 is associated with the presence of ERs and progesterone receptors 
in mammary and uterine cells $(10,11)$. The upregulation of H19 may be associated with the poor prognosis of TAM-resistant breast cancer, and the downregulation of H19 expression has been shown to inhibit the expression of transcription factors associated with the Wnt pathway and epithelial-mesenchymal transition (12). In addition, Basak et al (13) revealed that H19 upregulation was regulated by Notch and hepatocyte growth factor signaling in endocrine therapy-resistant cells, and that the inhibition of pathways regulating H19 expression significantly overcame TAM and fulvestrant resistance. These aforementioned findings have a certain significance for the present study.

While the mechanism by which $\mathrm{H} 19$ regulates transcription remains largely unknown, Tsang and Kwok (14) reported that $\mathrm{H} 19$ regulated multidrug resistance protein (MDR1) gene promoter methylation and induced MDR1-associated chemotherapy resistance in hepatocellular carcinoma cells. Thus, the alteration of target gene methylation may be a molecular mechanism underlying the regulation of gene transcription by H19.

\section{Materials and methods}

Cell culture and 4-hydroxytamoxifen treatment. MCF-7 breast cancer cells were obtained from the American Type Culture Collection and cultured as previously described (10). Briefly, MCF-7 cells were cultured in RPMI-1640 supplemented with $10 \%$ FBS (Biological Industries), $100 \mathrm{U} / \mathrm{ml}$ penicillin, $100 \mu \mathrm{g} / \mathrm{ml}$ streptomycin and $20 \mathrm{mM}$ L-glutamine (Invitrogen; Thermo Fisher Scientific, Inc.). TAM-resistant MCF-7 cells were developed by the treatment of wild-type MCF-7 cells with $1 \times 10^{-6}$ M 4-hydroxytamoxifen (Sigma-Aldrich; Merck KGaA) for 21 days and then $1 \times 10^{-7} \mathrm{M}$ 4-hydroxytamoxifen for 6 months, as described previously (15). All cells were maintained in a humidified incubator with $5 \% \mathrm{CO}_{2}$ at $37^{\circ} \mathrm{C}$. In the present study, 4-hydroxytamoxifen was used as it is the main active metabolite of TAM. These 4-hydroxytamoxifen-treated cells were named as MCF-7R and the corresponding parental cells as MCF-7.

Cell viability assay. To determine viable cell numbers, cells were plated in 96 -well plates $(5,000$ cells/well) and treated with either $0.1 \%$ dimethyl sulfoxide or 4-hydroxytamoxifen $\left(1 \times 10^{-10}\right.$, $1 \times 10^{-9}, 1 \times 10^{-8}, 1 \times 10^{-7}$ and $1 \times 10^{-6} \mathrm{M}$ ) for 24,48 and $72 \mathrm{~h}$. Cell viability was determined using an MTS assay and quantified by measuring the absorbance at $490 \mathrm{~nm}$. Cell viability is expressed as the fold of the corresponding control. The half-maximal inhibitory concentration $\left(\mathrm{IC}_{50}\right)$ of 4-hydroxytamoxifen for cell growth was determined by a dose-response experiment and calculated relative to the corresponding control.

Cell transfection and knockdown of gene expression by RNA interference. A synthetic RNA oligonucleotide targeting H19 was obtained from Guangzhou RiboBio Co., Ltd. H19 lncRNA was knocked down using a specific H19-targeting small interfering RNA (siRNA) and a short hairpin RNA (shRNA) vector. The H19 hairpin siRNA sequences were 5'-CATCAA AGACACCATCGGA-3'. The siRNA was subcloned into a pSilencer 2.1-U6 neovector (shRNAH19; Guangzhou RiboBio Co., Ltd.). A negative control siRNA and negative control
shRNA were also purchased from Guangzhou RiboBio Co., Ltd.

Briefly, cells were plated in phenol red-free medium containing 5\% stripped FBS (Biological Industries) in 12-well plates at a density of $\sim 70 \%$. Stripped FBS has been incubated with activated carbon that removes non-polar, lipophilic material, such as viruses, growth factors, hormones and cytokines regardless of molecular weight. This method has little effect on salts, glucose or amino acids. For the knockdown of H19, the cells were transfected with $200 \mathrm{nM}$ siRNA H19 for $24 \mathrm{~h}$ at $37^{\circ} \mathrm{C}$. Following knockdown, the cells were treated with 4-hydroxytamoxifen for $48 \mathrm{~h}$ at $37^{\circ} \mathrm{C}$ at the concentrations indicated in the respective assay. To generate a stable lncRNA H19 knockdown cell line, $1 \mu \mathrm{g}$ H19 shRNA was transfected into MCF-7R cells using Lipofectamine ${ }^{\circledR}$ RNAiMAX reagent (Invitrogen; Thermo Fisher Scientific, Inc.). The transfected cells were selected using G418 (Promega Corporation) and a stable clone was obtained and verified for H19 expression. All cells were maintained in a humidified incubator with $5 \% \mathrm{CO}_{2}$ at $37^{\circ} \mathrm{C}$. Following incubation for $48 \mathrm{~h}$, the transfected cells were harvested and utilized for subsequent experiments.

Breast cancer tissue sample collection. The current study included 30 primary premenopausal patients (age, 41.22 \pm 3.67 years) with ER-positive breast cancer. The patients underwent surgical resection at Xiangya Hospital (Changsha, China) and were randomly enrolled from January 2012 to December 2013. The resected tumor specimens were immediately frozen in liquid nitrogen and kept at $-80^{\circ} \mathrm{C}$ until RNA extraction. The collection and preservation of tumor and paired tumor adjacent tissues samples and the obtaining of written informed consent were approved by the Ethics Committee of Xiangya Hospital (project no. CTXY-140001-5). Patients continued to take $20-40 \mathrm{mg}$ tamoxifen daily following surgery for $\sim 1$ year. Regular follow-up, including clinical examination, chest X-ray and mammography, was performed for $\geq 5$ years.

Reverse transcription-quantitative-PCR (RT-qPCR). Total RNAs from harvested cells or patient tissues were prepared using TRIzol ${ }^{\circledR}$ reagent (Invitrogen; Thermo Fisher Scientific, Inc.) according to the manufacturer's protocol, and the RNA concentration was determined using a NanoDrop ${ }^{\mathrm{TM}} 2000$ spectrophotometer (Thermo Fisher Scientific, Inc.). The quality of the RNA samples was confirmed based on a ratio of absorbance values at 260 and $230 \mathrm{~nm}$ of $>1.7$ and a ratio of absorbance values at 260 and $280 \mathrm{~nm}$ of between 1.8 and 2.0. Total RNA (500 ng) was reversely transcribed to cDNA in a 20- $\mu 1$ reaction mixture comprising 200 units reverse transcriptase, 50 pmol random hexamer, 1X PCR buffer $[10 \mathrm{mM}$ Tris/ $\mathrm{HCl}$ (pH 9.0), $50 \mathrm{mM} \mathrm{KCl}$ and $1.5 \mathrm{mM} \mathrm{MgCl}_{2}$ ] and $1 \mathrm{mM}$ deoxynucleotide triphosphates (System Biosciences, LLC). The reaction products were diluted to a volume of $100 \mu \mathrm{l}$ with distilled water prior to qPCR. The qPCR mixture comprised $2 \mu \mathrm{l}$ diluted reverse transcription product, 1X SYBR-Green Master Mix (Applied Biosystems; Thermo Fisher Scientific, Inc.) and $50 \mathrm{nM}$ forward and reverse primers. qPCR was carried out using a LightCycler ${ }^{\circledR} 480$ Sequence Detection System (Roche Diagnostics). Following an initial 10-min incubation at $95^{\circ} \mathrm{C}$, thermocycling was carried out for 40 cycles of $95^{\circ} \mathrm{C}$ for $15 \mathrm{sec}$ and $60^{\circ} \mathrm{C}$ for $1 \mathrm{~min}$. Glyceraldehyde-3-phosphate 
dehydrogenase (GAPDH) was used as the reference gene. The primers used were as follows: H19 forward, 5'-GTCCGGCCT TCCTGAACACCTT-3' and reverse, 5'-GCTTCACCTTCC AGAGCCGAT-3' (10); NAT1 forward, 5'-AGCACTGGCATG ATTCACCTTCT-3' and reverse, 5'-GAGGCTGCCACATCT GGTAT-3' and GAPDH forward, 5'-TTGATTTTGGAGGGA TCTCGCTC-3' and reverse, 5'-GAGTCAACGGATTTGGTC GTATTG-3'. The level of RNA was expressed as a fold of the control calculated using the $2^{-\Delta \Delta \mathrm{Cq}}$ method (16).

Western blot analysis. Western blotting was conducted as previously described with minor modifications $(10,17)$. Briefly, cells were collected $48 \mathrm{~h}$ after the transfection with H19 siRNA, shRNA or the respective negative control to create lncRNA H19 knockdown cell lines. Total cellular proteins were extracted from the harvested cells using a lysis buffer (62.5 mM Tris- $\mathrm{HCl} \mathrm{pH} 6.8,100 \mathrm{mM}$ dithiothreitol, 2\% SDS and $10 \%$ glycerol). The protein concentrations were determined using the Bradford method with Bio-Rad Protein Assay reagent following the manufacturer's instructions (Bio-Rad Laboratories, Inc.). Then, $20 \mu \mathrm{g} /$ lane cell lysate was resolved using $12 \%$ sodium dodecyl sulfate polyacrylamide electrophoresis and transferred to nitrocellulose membranes. The blots were incubated in blocking buffer comprising 5\% non-fat dry milk in Tris-buffered saline with $0.5 \%$ Tween (TBS-T) at room temperature for $2 \mathrm{~h}$. After washing with TBS-T, the nitrocellulose membranes were incubated with a specific antibody against NAT1 (anti-rabbit; 1:250, cat. no. ab109114; Abcam) or $\beta$-actin (anti-mouse; 1:2,000; cat. no. AC-15; Sigma-Aldrich; Merck KGaA) overnight at $4^{\circ} \mathrm{C}$. Following the incubation with a horseradish peroxidase-conjugated secondary antibody (mouse anti-rabbit IgG; 1:10,000; cat. no. ab6728; Abcam) or rabbit anti-mouse IgG (1:10,000; cat. no. ab106762; Abcam) at room temperature for $1 \mathrm{~h}$, the signal was detected using an ECL Western Blotting system (Promega Corporation) and visualized using the ChemiDoc MP Imaging System (cat. no. 12003154; Bio-Rad Laboratories, Inc.). $\beta$-actin was used as the loading control.

Bisulfite genomic sequencing PCR (BSP) assay. Genomic DNA was isolated from the peripheral blood leucocytes with phenol-chloroform followed by ethanol precipitation. Chromosomal DNA from the MCF-7 and MCF-7R cells was treated with bisulfite using an EpiTect Bisulfite kit (Qiagen, Inc.). The methylation status of six $\mathrm{CpG}$ sites in the promoter region of the NAT1 gene was investigated (-790 to -1 of GenBank accession no. AY338489 in which the major transcriptional site is numbered +1) (8). A single ' $\mathrm{C}$ ' at the corresponding $\mathrm{CpG}$ site was considered as complete methylation, a single ' $\mathrm{T}$ ' as no methylation, and overlapping ' $\mathrm{C}$ ' and ' $\mathrm{T}$ ' as partial methylation. The gene-specific promoter regions were amplified via nested PCR from the bisulfite-treated DNA. The analysis of DNA methylation, including the primer sequences and PCR conditions used, was performed as previously described (8). The PCR products were purified by gel electrophoresis using $1 \%$ agarose gel, ligated into a pGEM-T plasmid (Promega Corporation) and transformed into DH5 $\alpha$ cells (Invitrogen; Thermo Fisher Scientific, Inc.) using standard heat-shock procedures (18). Blue/white screening was conducted and $\geq 10$ positive bacterial clones were sequenced as previously described (19). Quality control, sequence analysis and data illustration were performed using BiQ Analyzer 3.0 software (20). The data have been deposited in the CNGB Sequence Archive of the China National GeneBank DataBase with accession number CNP0001707.

Analysis of public breast cancer datasets. The importance of NAT1 in the outcome of patients with breast cancer treated with TAM was assessed by analyzing the relevance of NAT1 expression levels in breast cancer tissues to patient outcomes in the public datasets GSE2990 (21) and GSE20685 (22). R script-generated data with $\mathrm{P}<0.05$ from the GEO2R analysis of the GEO database (https://www.ncbi.nlm.nih. gov/geo/geo2r/) were obtained (23).

An online survival analysis tool was used to evaluate the effect of the NAT1 gene on breast cancer prognosis. Kaplan-Meier plots were generated using an online tool (http://kmplot.com/analysis/index.php? $\mathrm{p}=$ service $\&$ cancer $=$ breast $)(24)$.

Statistical analysis. Statistical analysis was performed using SPSS software (version 21.0; IBM Corp.). Each experiment was performed at least three times, and the data are presented as the mean \pm SEM. For parametric data, the Student's t-test was used to determine the statistical significance between two groups, and one-way ANOVA followed by a post hoc Tukey's test was used to compare differences among multiple groups. Kaplan-Meier plots for disease-free survival (DFS) were plotted and analyzed using log-rank tests. A total of 30 primary premenopausal patients were allocated to low and high H19 and NAT1 groups according to whether expression in the tumor tissue was $\geq 1.5$-fold lower or higher compared with paracancerous tissue. The fold difference of expression between tumor and adjacent normal tissue was $\geq 1.5$ in every case. Pearson correlation was used to measure the correlation between the expression levels of $\mathrm{H} 19$ and NAT1. P $<0.05$ was considered to indicate a statistically significant difference.

\section{Results}

Establishment of the MCF-7R cell line. To analyze the sensitivity of the MCF-7 and MCF-7R cell lines, the two cell lines were treated with different concentrations of 4-hydroxytamoxifen for 24-72 h. The viability of the MCF-7 cells was significantly inhibited by 4-hydroxytamoxifen at a concentration of $1 \times 10^{-7} \mathrm{M}$, while the viability of MCF-7R cells was significantly promoted (Fig. 1A). Furthermore, comparison of the MCF-7R and MCF-7 cells treated with $1 \times 10^{-7} \mathrm{M} 4$-hydroxytamoxifen reveals that the proliferation of the MCF-7R cells, as indicated by the viable cell number, was significantly increased at 48 and $72 \mathrm{~h}$ (Fig. 1B). These results suggest that MCF-7R cells have a significant resistance to 4-hydroxytamoxifen. Furthermore, 4-hydroxytamoxifen exhibited a dose- (Fig. 1A) and time-dependent (Fig. 1B) inhibitory effect on MCF-7 cells. The $\mathrm{IC}_{50}$ values were also calculated and were $148.1 \mathrm{nM}$ for the MCF-7 cells and $7.753 \mu \mathrm{M}$ for the MCF-7R cells (Fig. 1C).

Knockdown of IncRNA H19 restores TAM sensitivity in MCF-7R cells. As IncRNA H19 is an estrogen-inducible 

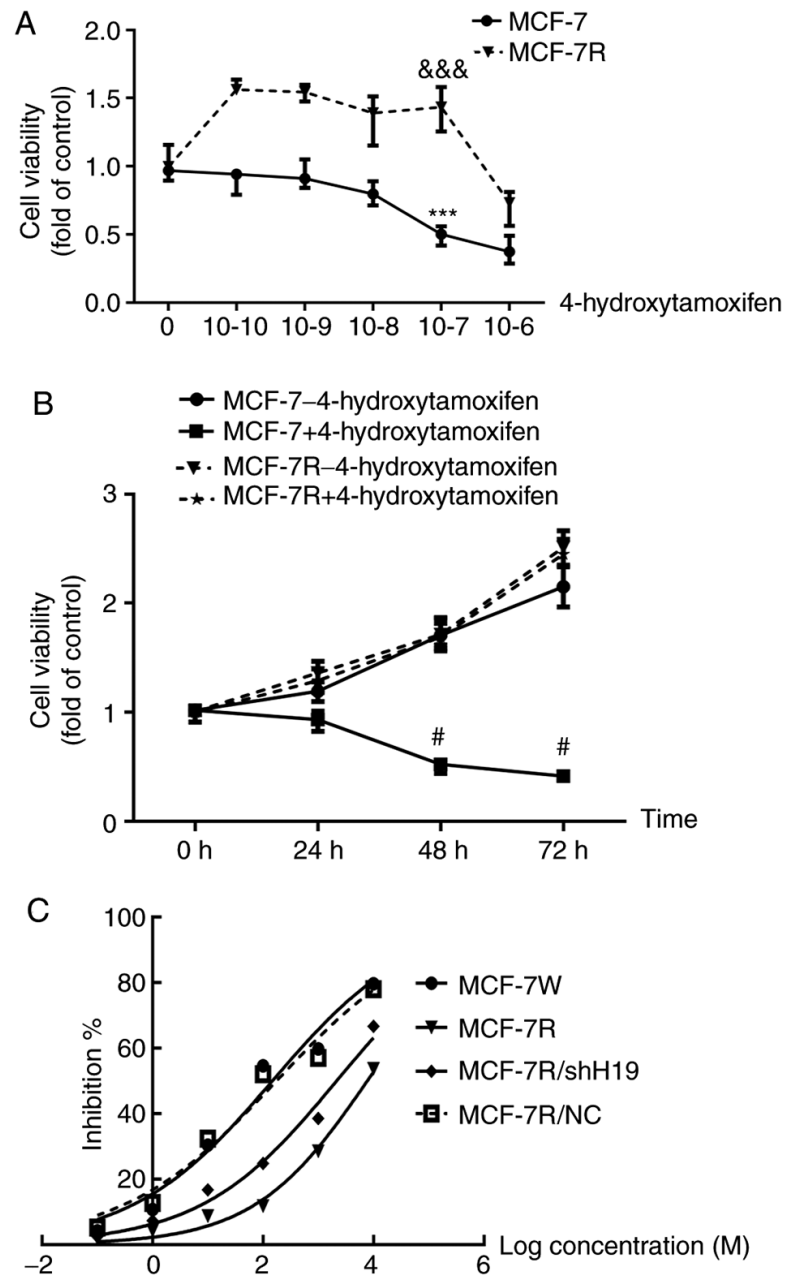

Figure 1. MCF-7 cells acquire TAM resistance as shown by increased cell viability. (A) MCF-7 and MCF-7R cells were cultured in phenol red-free RPMI-1640 with various concentrations of 4-hydroxytamoxifen for $48 \mathrm{~h}$. ${ }^{* * *} \mathrm{P}<0.001$ vs. the untreated control. ${ }^{\& \& \&} \mathrm{P}<0.001$ vs. the untreated control (B) MCF-7 and MCF-7R cells were treated with $1 \times 10^{-7} \mathrm{M}$ 4-hydroxytamoxifen for different durations. ${ }^{\text {}} \mathrm{P}<0.05$ vs. the $\mathrm{MCF}-7 \mathrm{R}+4$-hydroxytamoxifen group. (C) Different cell lines were treated with various concentrations of 4-hydroxytamoxifen for $48 \mathrm{~h}$, and the inhibition of cell viability was measured from which half-maximal inhibitory concentration values were calculated. Cell viability was determined using an MTS assay. Data are presented as the mean \pm SEM of three independent experiments. MCF-7R, TAM-resistant MCF-7 cells; TAM, tamoxifen; shH19, short hairpin RNA targeting H19; NC, negative control shRNA.

gene (10) and TAM is a partial ER agonist, it was hypothesized that IncRNA H19 expression may be elevated in MCF-7R cells. Therefore, the contribution of lncRNA H19 to the TAM resistance of MCF-7R breast cancer cells was investigated. The knockdown of lncRNA H19 in MCF-7R cells was conducted using H19-specific siRNA, which knocked down H19 lncRNA expression by $\sim 63 \%$ compared with that in the cells transfected with non-specific (NS) siRNA (Fig. 2A). Transfection with H19 siRNA significantly increased the sensitivity of the MCF-7R cells to 4-hydroxytamoxifen compared with that of the cells transfected with NS)siRNA (Fig. 2C). By contrast, transfection with NS siRNA, which did not affect H19 expression, had no effect on the sensitivity of the MCF-7R cells to 4-hydroxytamoxifen treatment.

To further investigate the function of lncRNA H19 in TAM-resistant cells, MCF-7R/shH19 cells were established by
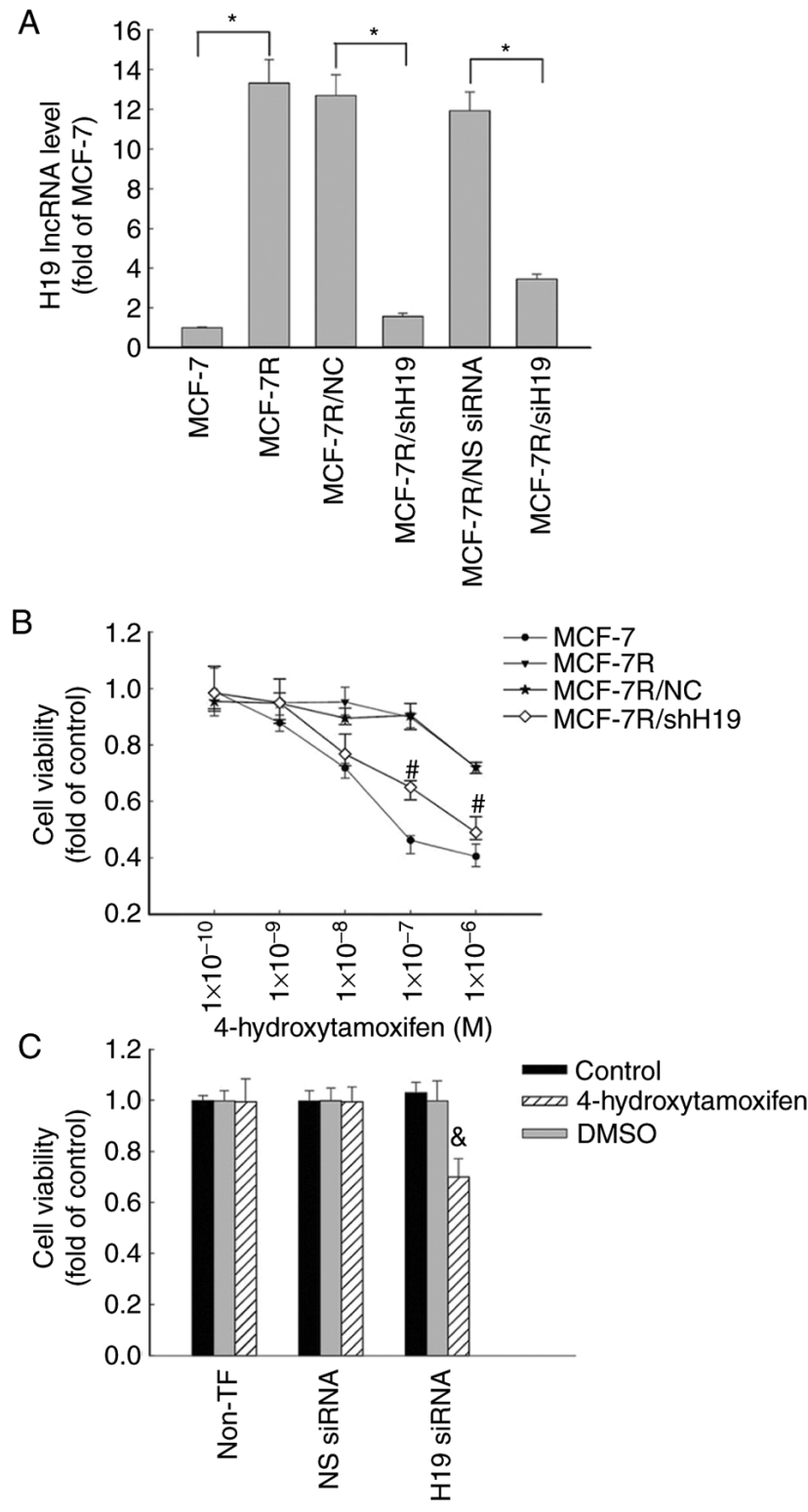

Figure 2. Increased expression of lncRNA H19 in MCF-7R cells is associated with tamoxifen resistance. (A) MCF-7R cells were transfected with siH19, NS siRNA, shH19 or NC shRNA. The levels of lncRNA H19 were quantified using reverse transcription-quantitative PCR in MCF-7R, H19-knockdown MCF-7R/siH19, MCF-7R/shH19 and corresponding control cells and are expressed as fold of that in the parental MCF-7 control. ${ }^{*} \mathrm{P}<0.05$ as indicated. (B) MCF-7, MCF-7R, MCF-7/NC and H19-knockdown MCF-7R/shH19 cells were plated in 96-well plates and treated with various concentrations of 4-hydroxytamoxifen for $48 \mathrm{~h}$ for the determination of cell proliferation using an MTS assay. ${ }^{~} \mathrm{P}<0.05$ vs. the MCF-7R/NC group. (C) Transfected MCF-7R/siH19 cells were treated with or without $1 \times 10^{-7} \mathrm{M}$ 4-hydroxytamoxifen for $48 \mathrm{~h}$ for the determination of cell proliferation using an MTS assay. All data are presented as the mean \pm SEM of three independent experiments. ${ }^{\circledR} \mathrm{P}<0.05$ vs. the NS siRNA/4-hydroxytamoxifen group. MCF-7R, tamoxifen-resistant MCF-7 cells; lncRNA, long non-coding RNA; siH19, siRNA targeting H19; NS, non-specific; siRNA, small interfering RNA; shH19, shRNA targeting H19; NC, negative control shRNA; shRNA, short hairpin RNA; non-TF, non-transfected.

the stable transfection of MCF-7R cells using a specific H19 shRNA expression vector to knock down H19 expression. The transfection reduced the expression of $\mathrm{H} 19$ lncRNA by $>90 \%$ in the MCF-7R/shH19 cells compared with the MCF-7R/NC cells, as revealed using RT-qPCR analysis. However, H19 
A

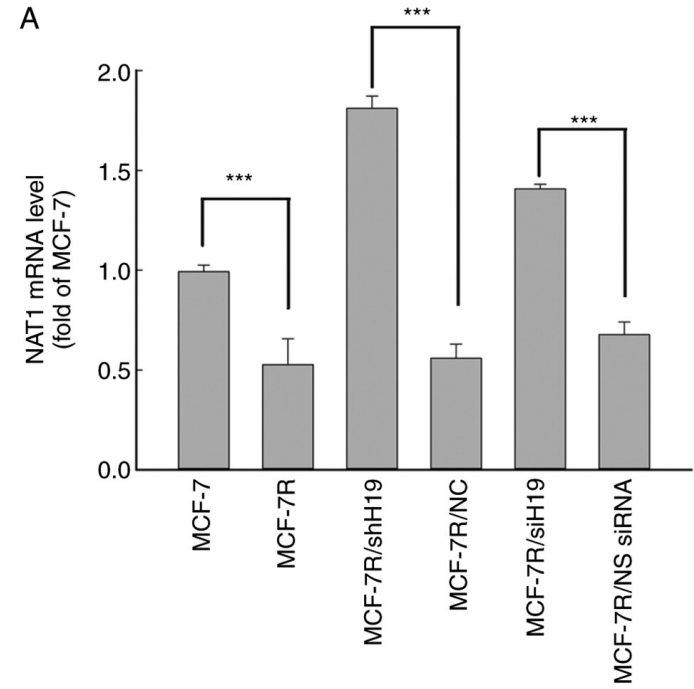

B

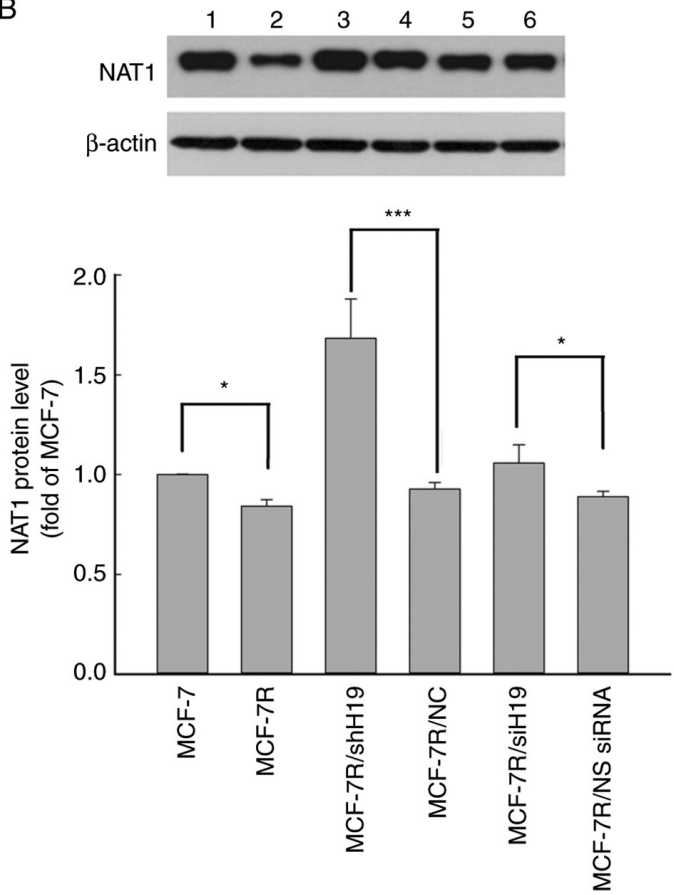

A

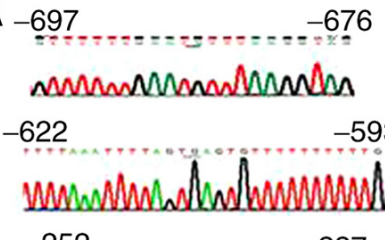

$-252$ $-227$

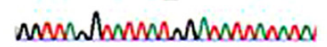

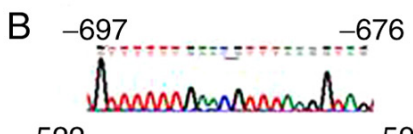

$-522$ $-593$

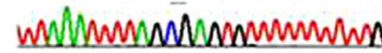

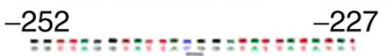

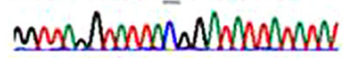

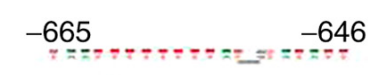

anararanannanma

$-316$ $-289$

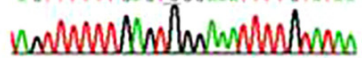

$-209$ $-182$

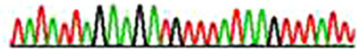

$-665$ $-639$

andmanumanmanul

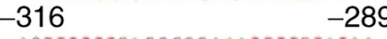
$-289$
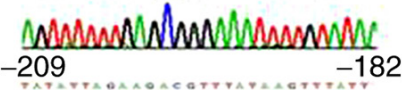

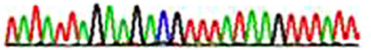

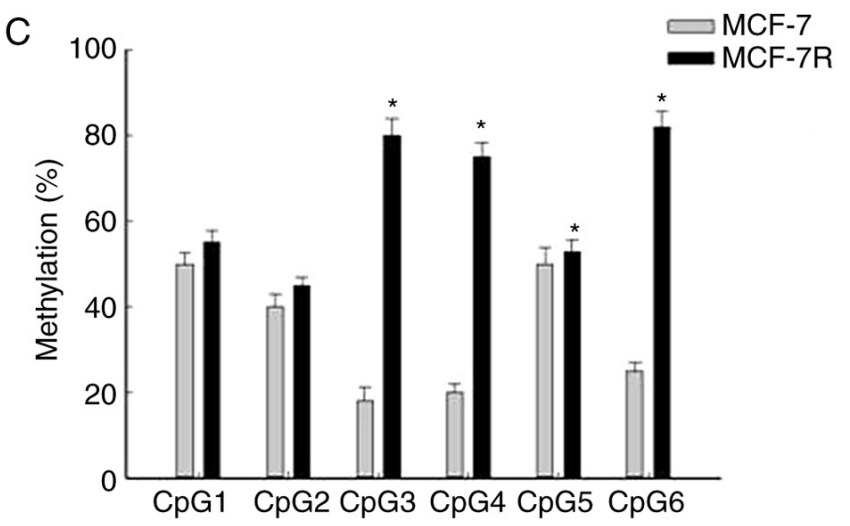

Figure 4. Methylation levels across the NAT1 promoter region. Representative DNA sequencing of (A) completely unmethylated and (B) completely methylated samples of the NAT1 gene. All unmethylated cytosines were changed to thymine by bisulfite treatment, but no methylated cytosines were changed. The CpG sites are indicated by underlining. (C) Methylation levels across the NAT1 promoter region. Data are presented as mean methylation of each CpG unit \pm standard deviation. Significant differences between the MCF-7 and MCF-7R cells were detected at $\mathrm{CpG} 3, \mathrm{CpG} 4, \mathrm{CpG} 5$ and $\mathrm{CpG} 6 .{ }^{*} \mathrm{P}<0.05$ vs. corresponding MCF-7. NAT1, N-acetyltransferase 1; MCF-7R, tamoxifen-resistant MCF-7 cells.

Figure 3. NAT1 expression is decreased in MCF-7R cells and rescued by long non-coding RNA H19 knockdown. NAT1 (A) mRNA and (B) protein expression levels were detected using reverse transcription-quantitative PCR and western blot analysis, respectively, in various cell lines. Representative blots are shown with lanes as follows: lane 1, MCF-7; lane 2, MCF-7R, lane 3, MCF-7R/shH19; lane 4, MCF-7/NC; lane 5, MCF-7R/siH19; lane 6, MCF-7R/NS siRNA. All data are presented as the mean \pm SEM of three independent experiments. ${ }^{*} \mathrm{P}<0.05$ and ${ }^{* * *} \mathrm{P}<0.05$ as indicated. NAT1, $\mathrm{N}$-acetyltransferase 1; MCF-7R, tamoxifen-resistant MCF-7 cells; siH19, siRNA targeting H19; NS, non-specific; siRNA, small interfering RNA; shH19, shRNA targeting H19; NC, negative control shRNA; shRNA, short hairpin RNA.

expression was not affected in the control vector transfected MCF-7R/NC cells (Fig. 2A). When lncRNA H19 was knocked down using shRNA, the MCF-7R cells were more sensitive to 4-hydroxytamoxifen therapy, with a significant decrease in cell viability (Fig. 2B) and a reduction in $\mathrm{IC}_{50}$ value from $7.753 \mu \mathrm{M}$ in the MCF-7R cells to $2.155 \mu \mathrm{M}$ in the MCF-7R/shH19 cells (Fig. 1C). These data suggest that IncRNA H19 may play a key role in the 4-hydroxytamoxifen resistance of MCF-7R cells.
NAT1 expression is decreased in MCF-7R cells and restored by lncRNA H19 knockdown. The mRNA and protein expression levels of NAT1 in MCF-7R cells were significantly lower compared with those in MCF-7 cells (Fig. 3). Following the knockdown of H19 in MCF-7R cells using H19 shRNA and siRNA, the mRNA and protein expression levels of NAT1 were significantly increased compared with those in the MCF-7R/NC and NS siRNA groups, respectively (Fig. 3). These results suggest that $\mathrm{H} 19$ serves an important role in TAM-resistant breast cancer cells via the regulation of NAT1 gene expression.

IncRNA H19 regulation of NAT1 gene promoter methylation in MCF-7R cells. The methylation status of the NAT1 gene promoter in MCF-7 and MCF-7R cells was examined using a BSP assay. Completely unmethylated (Fig. 4A) and completely methylated samples of the NAT1 gene were used (Fig. 4B). The methylation profiles of six CpG sites were investigated in the NAT1 upstream promoter region (8). The mean methylation levels in the MCF-7R group ranged from $45.18 \%$ at 
Table I. Methylation density of the NAT1 gene after knockdown of H19 in MCF-7 and MCF-7R cells.

\begin{tabular}{llcr}
\hline & \multicolumn{3}{c}{ Methylation density (\%) } \\
\cline { 2 - 4 } Cell line & Non-TF & NS siRNA & siRNA H19 \\
\hline MCF-7 & $30.78 \pm 2.2$ & $31.61 \pm 0.35$ & $36.5 \pm 2.92$ \\
MCF-7R & $66.11 \pm 0.75^{\mathrm{a}}$ & $63.84 \pm 2.02$ & $26.39 \pm 0.75^{\mathrm{b}}$ \\
\hline
\end{tabular}

MCF-7 and MCF-7R cells were transfected with siRNA H19 and the methylation status of the NAT1 gene promoter in the cells was examined using a bisulfite genomic sequencing PCR assay. Data were obtained from three separate experiments and are presented as the mean \pm SEM. A one-way ANOVA and Tukey's post hoc test were used for analysis. ${ }^{\mathrm{P}} \mathrm{P}<0.001 \mathrm{vs}$. MCF-7/non-TF; ${ }^{\mathrm{b}} \mathrm{P}<0.001$ vs. MCF-7R/non-TF. NAT1, N-acetyltransferase 1; MCF-7R, tamoxifen-resistant MCF-7 cells; non-TF, non-transfected; NS, non-specific; siRNA, small interfering RNA.

A

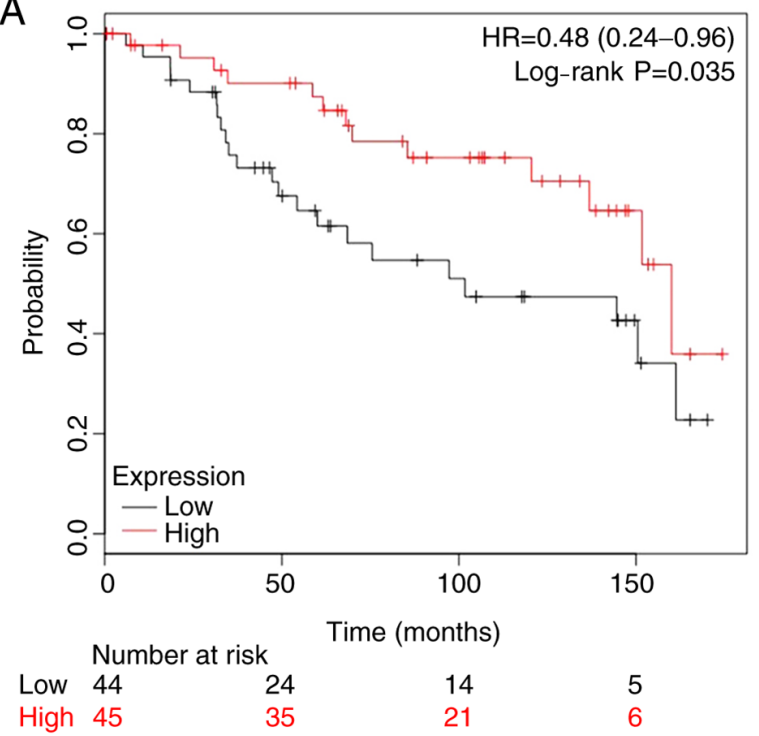

B

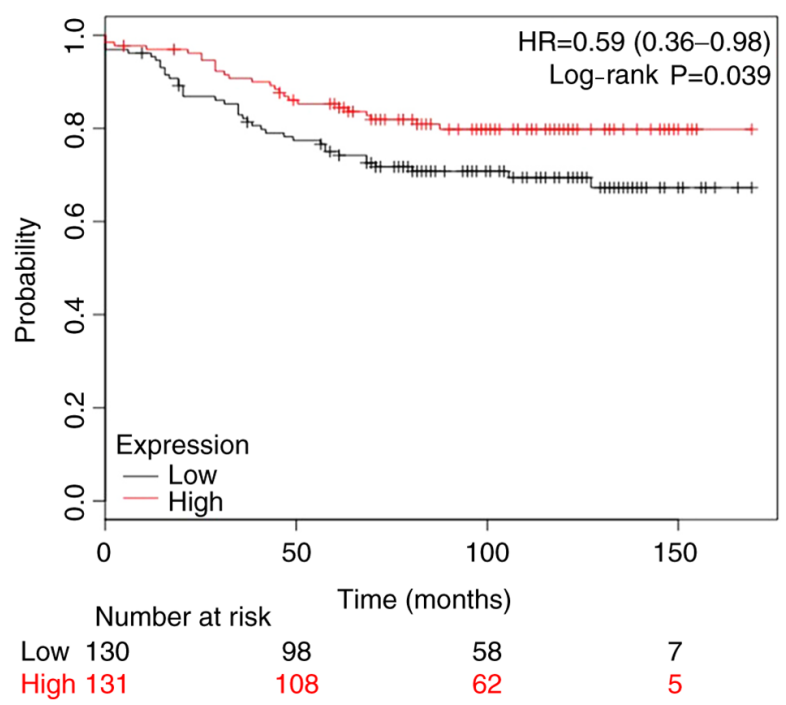

Figure 5. Low tumor NAT1 expression is associated with low survival in patients with breast cancer treated with TAM. Kaplan-Meier survival curve analysis was performed using tumor NAT1 expression levels (low or high) in two publicly available data sets for patients with estrogen receptor-positive breast cancer who received adjuvant TAM treatment. Survival curves based on mRNA expression data from (A) 89 patients and (B) 261 patients receiving TAM treatment. The red lines and numbers indicate high tumor NAT1 expression and the black lines and numbers indicate low tumor NAT1 expression. HR and log rank P-values were calculated based on data from the NCBI GEO database (accession numbers GSE2990 and GSE20685, respectively). HR, hazard ratio; NAT1, N-acetyltransferase 1; TAM, tamoxifen.

CpG2 to $82.17 \%$ at CpG6 (Fig. 4C). Comparison of NAT1 methylation profiles between the MCF-7 and MCF-7R groups indicated significant differences at $\mathrm{CpG} 3, \mathrm{CpG} 4, \mathrm{CpG} 5$ and CpG6 (Fig. 4C). The mean methylation rate of NAT1 in MCF-7R cells was significantly higher compared with that in MCF-7 cells (Table I). Moreover, H19 knockdown by siRNA transfection significantly decreased the mean methylation rate of the NAT1 promoter in MCF-7R cells compared with that in the untransfected cells (Table I). A higher methylation rate of NAT1 and decreased expression of NAT1 were observed in the MCF-7R group compared with the MCF-7 group, and H19 knockdown reversed these resistance-associated changes (Fig. 3 and Table I). This suggests that H19 may regulate NAT1 and drug resistance by altering the degree of NAT1 gene promoter methylation in MCF-7R cells.

Tumor NATI expression is associated with clinical outcome in patients with breast cancer treated with TAM. The present study analyzed two publicly available datasets, GSE2990 (n=89) and GSE20685 ( $\mathrm{n}=261)$, where patients with ER-positive breast cancer had been treated with adjuvant TAM monotherapy and $\geq 10$-year follow-up data were available. The Kaplan-Meier analysis demonstrated that low NAT1 expression was associated with significantly shorter overall survival compared with that of patients with high NAT1 expression (Fig. 5). With regard to the two datasets, they indicated that a higher mRNA expression level of NAT1 was favorable to the outcome of patients with breast cancer treated with TAM therapy.

Patient characteristics and responses. A total of 30 premenopausal patients with breast cancer who took TAM for at least 1 year after surgery were followed up for $\geq 5$ years. These patients were allocated to low and high H19 and NAT1 groups according to whether expression in the tumor tissue was $\geq 1.5$-fold lower or higher compared with paracancerous tissue. DFS was found to differ significantly according to the expression levels of H19 and NAT1. The DFS times of patients with high and low expression levels of H19 were 54.1 months 
A

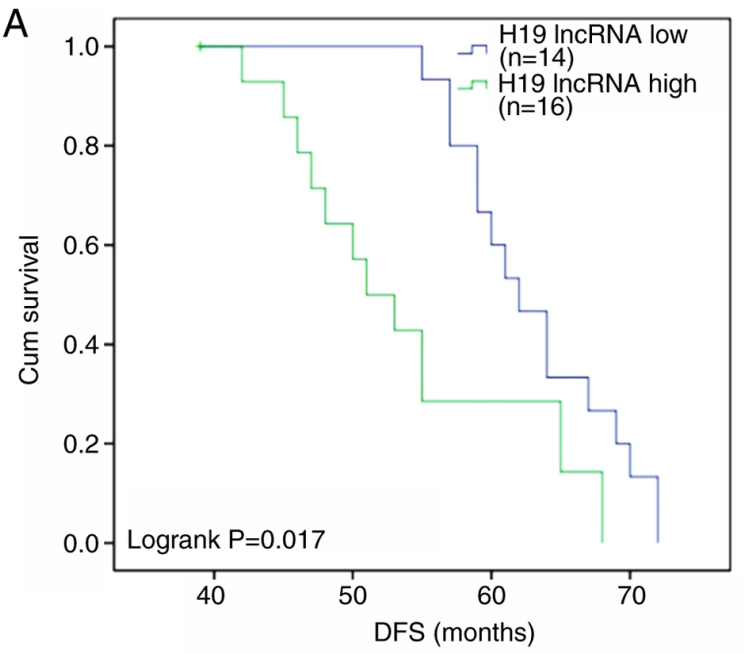

B

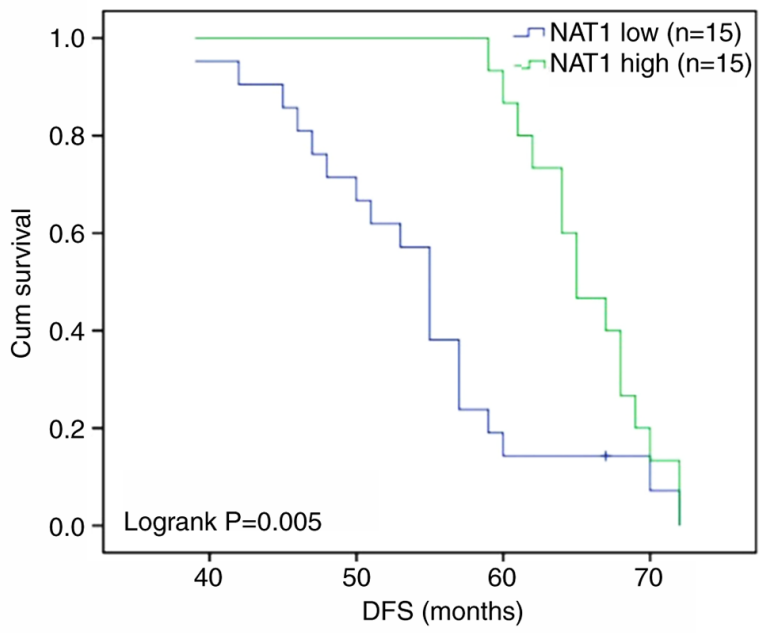

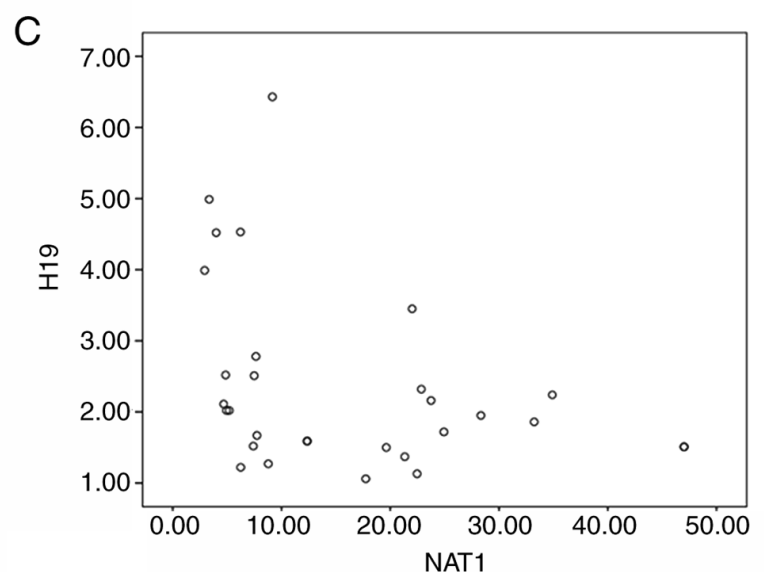

Figure 6. High expression levels of lncRNA H19 and low expression levels of NAT1 predict poor prognosis in patients with breast cancer receiving TAM therapy. Thirty patients were allocated to low and high $\mathrm{H} 19$ and NAT1 groups according to whether expression in the tumor tissue was $\geq 1.5$-fold lower or higher than that in the paracancerous tissue. Expression levels of H19 and NAT1 were detected using reverse transcription-quantitative PCR. (A) DFS of patients with TAM therapy in the low and high H19 expression groups. (B) DFS of patients with TAM therapy in the low and high NAT1 expression groups. (C) Pearson correlation analysis was used to measure the association between the expression levels of H19 and NAT1. IncRNA, long non-coding RNA; Cum, cumulative; DFS, disease-free survival; NAT1, N-acetyltransferase 1; TAM, tamoxifen.

(95\% CI, 49.5-58.8) and 63.2 months (95\% CI, 60.3-66.1), respectively (Fig. 6A), while the DFS times of patients with high and low expression levels of NAT1 were 65.7 months (95\% CI, 63.6-67.8) and 54.5 months (95\% CI, 50.7-58.3), respectively (Fig. 6B). Furthermore, a significant very weak negative correlation was identified between the expression levels of H19 and NAT1 in the individual samples (Pearson correlation coefficient, -0.0377; Fig. 6C). Collectively, these results suggest that higher expression of NAT1 and lower expression of H19 are associated with improved outcomes in patients with breast cancer treated with TAM.

\section{Discussion}

Although there have been advances in cancer therapy, TAM remains the mostly widely used endocrine therapy for patients with ER-positive breast cancer. However, $>40 \%$ of breast cancer cases develop resistance to endocrine therapy (4). Therefore, an improved understanding of the cellular and molecular pathways of TAM resistance may facilitate the development of strategies to overcome resistance.
Previous studies have reported that lncRNAs are a key component of gene regulatory networks and may serve a vital role in tumorigenesis and TAM resistance $(12,25,26)$. The results of the present study suggest that $\mathrm{H} 19$ is associated with TAM resistance in breast cancer. The modulating effect of $\mathrm{H} 19$ on NAT1 gene promoter methylation was further examined.

IncRNA H19 has been revealed to be an estrogen-regulated gene (10). Zhou et al (27) reported that H19 regulates epithelial-mesenchymal and mesenchymal-epithelial transition in breast cancer. The present study found that H19 expression was significantly upregulated in TAM-resistant (MCF-7R) cells, which is consistent with previous reports $(12,13)$. Moreover, the knockdown of 1ncRNA H19 in the MCF-7R cells ameliorated their resistance to 4-hydroxytamoxifen and promoted the inhibitory effect of 4-hydroxytamoxifen on cell proliferation; the knockdown of H19 enhanced the sensitivity to 4-hydroxytamoxifen in vitro. In a study by Wang et al (28), it was also reported that the knockdown of H19 significantly enhanced the sensitivity of TAM-resistant MCF7 cells to TAM in vitro and in vivo, and inhibited autophagy in these cells. It has also been observed that H19 expression is increased in doxorubicin 
(Dox)-resistant breast cancer (29). Furthermore, H19 upregulation has been observed in Dox-resistant liver cancer cells (14), TAM-resistant breast cancer cells, fulvestrant-resistant breast cancer cells $(13,28)$ and cisplatin-resistant lung cancer cells (30). Based on these findings, IncRNA H19 has potential as a useful biomarker and drug resistance target.

The present study demonstrated that NAT1 was significantly downregulated at the mRNA and protein levels in TAM-resistant cells. These results are consistent with previous reports that indicated the involvement of NAT1 in the TAM resistance of breast cancer cells $(8,9)$. The significance of NAT1 in TAM resistance was further demonstrated in the present study by the analysis of public breast cancer datasets, which indicated that low NAT1 expression was associated with poor survival in patients with breast cancer treated with TAM therapy. The analysis of primary patients also found that the high and low expression levels of NAT1 were associated with different DFS times and TAM response rates. In addition, NAT1 was upregulated at the mRNA and protein levels in TAM-resistant cells when H19 was knocked down, indicating that H19 controls NAT1 expression.

The present study investigated the molecular mechanism by which H19 modulates NAT1 gene expression; the methylation status of the NAT1 promoter in MCF-7 and MCF-7R cells was examined using the BSP method. The DNA methylation of NAT1 has been identified to serve a critical role in cancer, including breast cancer and colon adenocarcinoma $(9,31)$. Hypermethylation of the NAT1 gene may affect the initiation of TAM resistance (9). The results of the present study suggest that H19 may regulate NAT1 and thereby affect drug resistance by altering the degree of NAT1 promoter methylation in MCF-7R cells. The results also suggest that high H19 expression is associated with poor prognosis in patients with breast cancer treated with TAM therapy. Therefore, the present study indicates that H19 serves a crucial role in TAM resistance, which may facilitate the development of therapeutic strategies to ameliorate the resistance of cancer to endocrine therapy. However, it must be noted the sample size is the present study is small, which is a limitation, and a larger sample size is required in future studies to confirm the results.

Therefore, the present study suggests that the H19 gene regulates NAT1 expression in TAM-resistant cells via the mediation of NAT1 promoter methylation. Further elucidation of the role of H19 in the modulation of NAT1 gene expression and methylation should improve our understanding of endocrine therapy resistance, and may provide a theoretical basis for further studies evaluating targeted drugs.

\section{Acknowledgements}

Not applicable.

\section{Funding}

This work was supported in part by grants from the National Natural Science Foundation of China (grant nos. 81673516 and 81403021), Natural Science Foundation of Fujian (grant no. 2019J01177), Fujian Science and Technology Innovation Joint Fund Project (grant no. 2017Y9067), High-level hospital foster grants from Fujian Provincial Hospital (grant no. 2019HSJJ06), Fujian Province Young and Middle-aged
Talent Training Program (grant no. 2019-ZQN-35) and the Medical Science Research Project (grant no. BJBQEKYJJ-B19001CS).

\section{Availability of data and materials}

The datasets used and/or analyzed during the current study are available from the corresponding author on reasonable request. The BSP data have been deposited into the CNGB Sequence Archive of the China National GeneBank DataBase with accession number CNP0001707.

\section{Authors' contributions}

HS, GW and JZ participated in research design. HS, YZ and YP conducted experiments. HS, JC, XW performed data analysis. HS, JC, GW wrote or contributed to the writing of the manuscript. HS and GW confirm the authenticity of all the raw data. All authors read and approved the final manuscript.

\section{Ethics approval and consent to participate}

The study was approved by the Ethics Committee of Xiangya Hospital (project no. CTXY-140001-5). Written informed consent was obtained from all patients.

\section{Patient consent for publication}

The patients who participated in this study provided signed consent for publication.

\section{Competing interests}

The authors declare that they have no competing interests.

\section{References}

1. Bray F, Ferlay J, Soerjomataram I, Siegel RL, Torre LA and Jemal A: Global cancer statistics 2018: GLOBOCAN estimates of incidence and mortality worldwide for 36 cancers in 185 countries. CA Cancer J Clin 68: 394-424, 2018.

2. Harvey JM, Clark GM, Osborne CK and Allred DC: Estrogen receptor status by immunohistochemistry is superior to the ligand-binding assay for predicting response to adjuvant endocrine therapy in breast cancer. J Clin Oncol 17: 1474-1481, 1999.

3. Drăgănescu M and Carmocan C: Hormone therapy in breast cancer. Chirurgia (Bucur) 112: 413-417, 2017.

4. Ring A and Dowsett M: Mechanisms of tamoxifen resistance. Endocr Relat Cancer 11: 643-658, 2014.

5. Abdel-Hafiz HA: Epigenetic mechanisms of tamoxifen resistance in luminal breast cancer. Diseases 5: 16, 2017.

6. Lo PK and Sukumar S: Epigenomics and breast cancer. Pharmacogenomics 9: 1789-1902, 2008

7. Kim SJ, Kang HS, Chang HL, Jung YC, Sim HB, Lee KS, Ro J and Lee ES: Promoter hypomethylation of the $\mathrm{N}$-acetyltransferase 1 gene in breast cancer. Oncol Rep 19: 663-668, 2008.

8. Kim SJ, Kang HS, Jung SY, Min SY, Lee S, Kim SW, Kwon Y, Lee KS, Shin KH and Ro J: Methylation patterns of genes coding for drug-metabolizing enzymes in tamoxifen-resistant breast cancer tissues. J Mol Med (Berl) 88: 1123-1131, 2010.

9. Berteaux N, Lottin S, Monté D, Pinte S, Quatannens B, Coll J, Hondermarck H, Curgy JJ, Dugimont T and Adriaenssens E: H19 mRNA-like noncoding RNA promotes breast cancer cell proliferation through positive control by E2F1. J Biol Chem 280: 29625-29636, 2015. 
10. Sun H, Wang G, Peng Y,Zeng Y,Zhu QN, Li TL, Cai JQ, Zhou HH and Zhu YS: H19 lncRNA mediates $17 \beta$-estradiol-induced cell proliferation in MCF-7 breast cancer cells. Oncol Rep 33: 3045-3052, 2015.

11. Adriaenssens E, Lottin S, Dugimont T, Fauquette W, Coll J, Dupouy JP, Boilly B and Curgy JJ: Steroid hormones modulate $\mathrm{H} 19$ gene expression in both mammary gland and uterus. Oncogene 18: 4460-4473, 1999.

12. Gao H, Hao G, Sun Y, Li L and Wang Y: Long noncoding RNA H19 mediated the chemosensitivity of breast cancer cells via Wnt pathway and EMT process. Onco Targets Ther 11: 8001-8012, 2018.

13. Basak P, Chatterjee S, Bhat V, Su A, Jin H, Lee-Wing V, Liu Q, Hu P, Murphy LC and Raouf A: Long non-coding RNA H19 acts as an estrogen receptor modulator that is required for endocrine therapy resistance in ER+ breast cancer cells. Cell Physiol Biochem 51: 1518-1532, 2018.

14. Tsang WP and Kwok TT: Riboregulator H19 induction of MDR1-associated drug resistance in human hepatocellular carcinoma cells. Oncogene 26: 4877-4881, 2007.

15. Coser KR, Wittner BS, Rosenthal NF, Collins SC, Melas A, Smith SL, Mahoney CJ, Shioda K, Isselbacher KJ, Ramaswamy S and Shioda T: Antiestrogen-resistant subclones of MCF-7 human breast cancer cells are derived from a common monoclonal drug-resistant progenitor. Proc Natl Acad Sci USA 106: 14536-14541, 2009.

16. Livak KJ and Schmittgen TD: Analysis of relative gene expression data using real-time quantitative PCR and the 2(-Delta Delta C(T)) method. Methods 25: 402-408, 2001.

17. Butcher NJ, Ilett KF and Minchin RF: Substrate-dependent regulation of human Arylamine N-Acetyltransferase-1 in cultured cells. Mol Pharmacol 57: 468-473, 2000.

18. Froger A and Hall JE: Transformation of plasmid DNA into E. coli using the heat shock method. J Vis Exp: Aug 1, 2007 (Epub ahead of print). doi: 10.3791/253.

19. Reed K, Hembruff SL, Laberge ML, Villeneuve DJ, Côté GB and Parissenti AM: Hypermethylation of the ABCB1 downstream gene promoter accompanies ABCB1 gene amplification and increased expression in docetaxel-resistant MCF-7 breast tumor cells. Epigenetics 3: 270-280, 2008.

20. Bock C, Reither S, Mikeska T,Paulsen M, Walter J and Lengauer T: BiQ Analyzer: Visualization and quality control for DNA methylation data from bisulfite sequencing. Bioinformatics 21: 4067-4068, 2005.

21. Jiang X, Menon A, Wang S, Kim J and Ohno-Machado L: Doubly optimized calibrated support vector machine (DOC-SVM): An algorithm for joint optimization of discrimination and calibration. PLoS One 7: e48823, 2012.
22. Meng L, Xu Y, Xu C and Zhang W: Biomarker discovery to improve prediction of breast cancer survival: Using gene expression profiling, meta-analysis, and tissue validation. Onco Targets Ther 9: 6177-6185, 2016

23. Barrett T, Wilhite SE, Ledoux P, Evangelista C, Kim IF, Tomashevsky M, Marshall KA, Phillippy KH, Sherman PM, Holko M, et al: NCBI GEO: Archive for functional genomics data sets-update. Nucleic Acids Res 41 (Database Issue): D991-D995, 2013.

24. Pénzváltó Z, Lánczky A, Lénárt J, Meggyesházi N, Krenács T, Szoboszlai N, Denkert C, Pete I and Győrffy B: MEK1 is associated with carboplatin resistance and is a prognostic biomarker in epithelial ovarian cancer. BMC Cancer 14: 837, 2014.

25. Xue X, Yang YA, Zhang A, Fong KW, Kim J, Song B, Li S, Zhao JC and Yu J: lncRNA HOTAIR enhances ER signaling and confers tamoxifen resistance in breast cancer. Oncogene 35: 2746-2755, 2016.

26. Teunissen SF, Jager NG, Rosing H, Schinkel AH, Schellens JH and Beijnen $\mathrm{JH}$ : Development and validation of a quantitative assay for the determination of tamoxifen and its five main phase I metabolites in human serum using liquid chromatography coupled with tandem mass spectrometry. J Chromatogr B Analyt Technol Biomed Life Sci 879: 1677-1685, 2011.

27. Zhou W, Ye XL, Xu J, Cao MG, Fang ZY, Li LY, Guan GH, Liu Q, Qian YH and Xie D: The lncRNA H19 mediates breast cancer cell plasticity during EMT and MET plasticity by differentially sponging miR-200b/c and let-7b. Sci Signal 10: eaak9557, 2017.

28. Wang J, Xie S, Yang J, Xiong H, Jia Y, Zhou Y, Chen Y, Ying X, Chen $\mathrm{C}$, Ye C, et al: The long noncoding RNA H19 promotes tamoxifen resistance in breast cancer via autophagy. J Hematol Oncol 12: 81, 2019.

29. Zhu QN, Wang G, Guo Y, Peng Y, Zhang R, Deng JL, Li ZX and Zhu YS: lncRNA H19 is a major mediator of doxorubicin chemoresistance in breast cancer cells through a cullin4A-MDR1 pathway. Oncotarget 8: 91990-92003, 2017.

30. Wang Q, Cheng N, Li X, Pan H, Li C, Ren S, Su C, Cai W, Zhao C, Zhang L and Zhou C: Correlation of long non-coding RNA H19 expression with cisplatin-resistance and clinical outcome in lung adenocarcinoma. Oncotarget 8: 2558-2567, 2017.

31. Shi C, Xie LY, Tang YP, Long L, Li JL, Hu BL and Li KZ: Hypermethylation of $\mathrm{N}$-Acetyltransferase 1 is a prognostic biomarker in colon adenocarcinoma. Front Genet 10: 1097, 2019.

c) (i) $\odot$ This work is licensed under a Creative Commons Attribution-NonCommercial-NoDerivatives 4.0 International (CC BY-NC-ND 4.0) License. 